\title{
Psychosis research at Maastricht University,
}

\section{The Netherlands}

JIM VAN OS and PHILIPPE DELESPAUL

The academic department of psychiatry at Maastricht University is situated not only in the university hospital, but crucially also in nearly all the affiliated mental health institutions in a region with a source population of 650000 . This set-up is funded by a central government grant, allowing academic personnel to work in affiliated mental health institutions. Research projects are carried out on the basis of 4-year PhD contracts, or, in the case of doctors with specialist training, through research psychiatrist appointments in the regional academic psychiatric network. Strong links exist with the Institute of Psychiatry in London.

\section{WORK PERFORMED AND ONGOING}

\section{Population variation of psychiatric phenotypes}

Several large-scale sampling frames have been used to examine dimensional phenotypes of psychosis. In joint $\mathrm{PhD}$ projects with the Netherlands Institute for Mental Health, around 7000 individuals who had been randomly sampled from the general population were investigated repeatedly for attenuated, non-clinical forms of psychosis over a period of 3 years. Up to a fifth of people in this sample displayed psychotic experiences along dimensions of positive and negative symptoms. In order to investigate possible continuity between these experiences and DSM-diagnosed psychotic disorder, several analytical approaches were developed. First, continuity was shown between the non-clinical and clinical expressions of psychosis in terms of key demographic associations such as age, gender and marital status. Second, longitudinal continuity was shown, in that with time a substantial proportion of the sample changed status from non-clinical to clinical or vice versa. Third, a degree of aetiological continuity was shown, in that variably exposed populations always displayed a directionally and quantitatively similar change in both the clinical and non-clinical expressions of psychosis, suggesting that their prevalences are subject to the same underlying aetiological force.

These basic findings are now under further investigation using several approaches. The first is to collect large population-based samples of (a) extended families and (b) twin pairs to investigate patterns of familial clustering of dimensions of psychosis, and to examine covariation with known psychosis endophenotypes, in particular in the realm of cognition. Instruments to screen for experience of psychosis in the general population, such as the Community Assessment of Psychic Experiences (CAPE; Stefanis et al, 2002), have been developed in collaboration with Hélène Verdoux from the University of Bordeaux and Nick Stefanis from the Institute of Psychiatry in Athens. Early results suggest that dimensions of psychosis in the general population are distributed and covary with measures of cognition. In addition, part of this pattern of covariation is genetic and may constitute a suitable quantitative phenotype of psychosis for molecular genetic studies. The second approach is to investigate population samples that are at critical maturational stages with respect to onset of psychosis. In joint $\mathrm{PhD}$ projects with Hans-Ulrich Wittchen and Roselind Lieb at the Max Planck Institute in Munich, the onset and course of psychosis in a population sample of around 3000 adolescents are being investigated. This sample provides the opportunity to examine at the population level how psychosis is expressed in adolescence and, critically, whether the gender differences in age at onset seen in schizophrenia also apply to the broader phenotype of non-clinical psychotic experiences, which would suggest that a normal, gender-related maturational event after puberty causes expression of psychosis along a continuum of severity (Spauwen et al, 2003).

\section{Ecological and genetic interactions}

Several approaches are used to unravel the gene-environment and environmentenvironment interactions that are thought to drive variation of (risk for) psychosis (Jacobs et al, 2002; Wichers et al, 2002; van Os et al, 2003). A powerful approach to studying the influence of the wider social environment is to determine environmental influences that are shared by people within ecological units such as cities and, within these, neighbourhoods. As the risk of psychosis is shaped over the life course, the hypothesis is investigated that the wider social environment might contribute to the risks threatening later mental health by affecting the development of children. To this end, cohorts of children in the general population were followed in the neighbourhoods of Maastricht and Rotterdam. The first results indicate that mental health and deviant behaviour in children are specifically associated with one aspect of neighbourhood social capital, namely the degree of informal social control (Drukker et al, 2003). These findings should be interpreted in the light of the finding that the variation of schizophrenia across Maastricht neighbourhoods is not random: analyses linking this variation to neighbourhood social capital have been carried out. In order to examine to what degree the wider social environment affects the risk for psychotic disorder together with genetic influences, cohort data from The Netherlands and data from the Danish National Case Register are being analysed in collaboration with Carsten Pedersen and Preben Mortensen. The results of the analyses in both data-sets suggest that risk for schizophrenia is, in part, synergistically shaped by the proxy environmental risk factor urbanicity and the proxy genetic risk factor of having a first-degree relative with psychosis.

In addition to effects of the wider social environment, the influence of the microenvironment is being studied in a variety of ways. The 'experience-sampling method', which randomly samples experience of environmental exposures and their mental health effects in the flow of daily life, has been used to show that individuals with psychotic disorder had increased emotional reactivity to stressors occurring in the flow of daily life and that their first-degree relatives had emotional reactivity levels intermediate between those of patients and well controls. Follow-up studies suggested that emotional reactivity to daily life stress 
also played a part in depressive and bipolar disorders and that it was independent of the cognitive impairments seen in patients, although it might be associated with brain volumetric measures (Marcelis et al, 2003). The presence of environmentenvironment interactions was suggested by the finding that prior exposure to major life events increased the subsequent level of emotional reactivity (Myin-Germeys et al, 2003). Further experience-sampling method studies are underway to investigate the influence of dopamine-releasing drugs such as cannabis on individuals at risk of psychotic disorder, combined with challenge studies involving cannabis and metabolic stress. A longitudinal study of around 300 twin pairs is currently investigating in more detail the significance of emotional reactivity assessed using the experience-sampling method, and its impact on psychopathology. By coupling the random sampling of experiences in daily life with salivary cortisol measurements, the interplay between emotional reactivity to environmental exposures and hypothalamic-pituitaryadrenal axis reactivity can be assessed, while the twin design will allow quantification of the relative contribution of genes and environment to these processes in daily life. Concurrent assessment of personality characteristics, in particular neuroticism, will help to clarify the role of neuroticism in shaping the risk not only of depression, but also of psychosis.

\section{Cognitive epidemiology}

The impact of any environmental exposure on psychosis risk is thought to be mediated by cognitive processes involving causal attributions, emotional appraisals and cognitive biases that might facilitate the onset of delusions and hallucinations. Current cognitive models of psychosis thus provide hypotheses about the role of social and personality factors in shaping cognitive vulnerabilities to psychosis. Some aspects of these models can be readily tested using traditional epidemiological methods, in particular the cohort study (Janssen et al, 2003). In a longitudinal cohort study of 80 adolescent participants who were hearing voices, it was found that those who needed mental health care reported more perceived influence of the voices on feelings and behaviour, and more negative affective appraisals in relation to the voices. In

JIM VAN OS, PhD, Department of Psychiatry and Neuropsychology, Maastricht University, The Netherlands, and Division of Psychological Medicine, Institute of Psychiatry, London, UK; PHILIPPE DELESPAUL, PhD, Department of Psychiatry and Neuropsychology, Maastricht University, The Netherlands

Correspondence: Professor J. van Os, Department of Psychiatry and Neuropsychology, PO Box 616 (DRT I0), 6200 MD Maastricht, The Netherlands. Tel: 43 3875443; fax: 43 3875444; e-mail: j.vanos@sp.unimaas.nl

addition, it was found that formation of delusional ideation over the follow-up period was related to voice attributions associated with external sources, authority figures, perceived influence or 'power' over the person, as well as emotional appraisal processes (Escher et al, 2002). In the Netherlands Mental Health Survey and Incidence Study (NEMESIS), it was shown that in the general population non-clinical experience of hallucinations was more likely to result in need for mental health care over a 3 -year period if the person developed depression and delusional interpretations (Krabbendam et al, 2003). In addition, it was shown that individuals with a symptomatic coping style (going along with and indulging in the experience of psychosis) were more likely to experience loss of control and develop need for care (Bak et al, 2003).

\section{NEW INITIATIVES AND CHALLENGES FOR THE FUTURE}

The cognitive mechanisms discussed above are being examined in relation to measures of social cognition in longitudinal studies of both patients and individuals at risk. For example, in a collaborative $\mathrm{PhD}$ project with Richard Bentall from Manchester University, the experience-sampling method will be used to examine the relationship between self-esteem and paranoia in the flow of daily life. In a national collaborative study with the universities of Amsterdam, Groningen, Maastricht and Utrecht, a national sample of about 1000 individuals with psychosis, as well as their parents and their siblings, will be followed over a period of 10 years in order to examine, among other things, cognitive processes in relation to onset and course of psychosis.

\section{ACKNOWLEDGEMENTS}

The senior investigators of the Maastricht Psychosis Programme are Inez Germeys, Lydia Krabbendam, Machteld Marcelis, Maarten Bak, Joost à Campo, Nancy Nicolson, Philippe Delespaul and Jim van Os.

\section{REFERENCES}

Bak, M., Hanssen, M., Bijl, R.V., et al (2003) When does experience of psychosis result in need for care? A prospective general population study. Schizophrenia Bulletin, in press.

Drukker, M., Kaplan, C., Feron, F., et al (2003) Children's health-related quality of life, neighbourhood socio-economic deprivation and social capital. A contextual analysis. Social Science and Medicine, 57, 825-841.

Escher, S., Romme, M., Buiks, A., et al (2002) Formation of delusional ideation in adolescents hearing voices: a prospective study. American Journal of Medical Genetics, II4, 913-920.

Jacobs, N., Rijsdijk, F., Derom, C., et al (2002) Child psychopathology and lower cognitive ability: a general population twin study of the causes of association. Molecular Psychiatry, 7, 368-374.

Janssen, I., Hanssen, M., Bak, M., et al (2003) Discrimination and delusional ideation. British Journal of Psychiatry, 182, 7I-76.

Krabbendam, L., Hanssen, M., Bak, M., et al (2003) Psychotic features in the general population. Risk factors for what? In Search for the Causes of Schizophrenia (eds W. F. Gattaz \& H. Hafner). Berlin: Springer Verlag.

Marcelis, M., Myin-Germeys, I., Suckling, J., et al (2003) Cerebral tissue alterations and daily life stress experience in psychosis. Acta Psychiatrica Scandinavica, 107, 54-59.

Myin-Germeys, I., Krabbendam, L., Delespaul, P. A., et al (2003) Do life events have their effect on psychosis by influencing the emotional reactivity to daily life stress? Psychological Medicine, 33, 327-333.

Spauwen, J., Krabbendam, L., Lieb, R., et al (2003) Sex differences in psychosis: normal or pathological? Schizophrenia Research, 62, 45-49.

Stefanis, N. C., Hanssen, M., Smirnis, N. K., et al (2002) Evidence that three dimensions of psychosis have a distribution in the general population. Psychological Medicine, 32, 347-358.

Van Os, J., Hanssen, M., Bak, M., et al (2003) Do urbanicity and familial liability coparticipate in causing psychosis? American Journal of Psychiatry, 160, 477-482.

Wichers, M. C., Purcell, S., Danckaerts, M., et al (2002) Prenatal life and post-natal psychopathology: evidence for negative gene-birth weight interaction. Psychological Medicine, 32, 1165-1174. 